\section{Manejo estomatológico del paciente pediátrico con hemofilia: reporte de casos.}

\section{Stomatologic management of pediatric patients with hemophilia: Case report.}

\author{
Avendaño Córdova, Karla Beatriz ${ }^{1 *}$ \\ https://orcid.org/0000-0002-1386-4946
}

\author{
Marco Antonio Rueda Ventura 1 \\ https://orcid.org/0000-0001-8306-0428
}

Laura Beatriz Isidro Olán 1

https://orcid.org/0000-0002-2944-0558

1 Universidad Juárez Autónoma de Tabasco. *Correspondencia: karlitab95@hotmail.com

DOI: $10.19136 / \mathrm{mhr} . a 5 n 1.4385$

\begin{abstract}
Resumen
La hemofilia es un trastorno genético que altera los factores de coagulación, en el cuál, al no coagular la sangre de manera correcta, puede causar una hemorragia espontánea. No existe cura para erradicar este padecimiento. Sin embargo, los profesionales del área de salud han elaborado guías de práctica clínica y manuales para el manejo de pacientes con trastornos de la coagulación con integración multidisciplinaria para realizar tratamientos preventivos y curativos, donde plantean la administración de terapia de remplazo juntamente con medidas alternas de hemostasia en el tratamiento odontológico, reduciendo los riesgos de caries múltiples y enfermedades periodontales.

El propósito de este trabajo fue describir el manejo odontológico de pacientes con Hemofilia a través de una serie de casos, donde se pudo realizar procedimientos dentales preventivos y restaurativos empleando medidas alternas de hemostasia y reduciendo la administración de terapia de remplazo.
\end{abstract}

Palabras clave: Hemofilia, terapia de remplazo, hemostasia, caries.

\begin{abstract}
Hemophilia is a genetic disorder that alters the coagulation factors, in which by not coagulating the blood correctly, it can cause spontaneous bleeding. There is no cure to eradicate this condition. However, health professionals have developed clinical practice guides and manuals for the management of patients with coagulation disorders with multidisciplinary integration to perform preventive and curative treatments, where they propose the administration of replacement therapy together with alternative measures of hemostasis in dental treatment, reducing the risks of multiple caries and periodontal diseases. The purpose of this work was to describe the dental management of patients with hemophilia through a series of cases, where preventive and restorative dental procedures could be performed using alternative measures of hemostasis and reducing the administration of replacement therapy.
\end{abstract}

Key words: hemophilia, replacement therapy, hemostasis, dental cavities. 


\section{Introducción}

La Hemofilia es una enfermedad que afecta a un gen del cromosoma $X$ que altera los factores de coagulación. Las mujeres son las portadoras de Hemofilia y los hombres la padecen, esta se clasifica de acuerdo con el déficit de factor circulante en sangre en: Hemofilia A, deficiencia de factor VIII, Hemofilia B deficiencia de factor IX y Hemofilia C, deficiencia de factor XI. Con base en la gravedad se clasifica en leve (5 a $>40 \%)$, moderada ( 1 a $5 \%$ ) y severa $(<1 \%)$. Un signo identificable en los pacientes es la tendencia a hemorragias internas, músculos, mucosa oral, nariz, tracto genitourinario, sangrados intracraneales y garganta causada por traumatismos o procedimientos (Brewer et al., 2012).

La Federación Mundial de Hemofilia (Brewer et al., 2012) menciona que aproximadamente. 1 de cada 10.000 personas nace con esta enfermedad. Estos pacientes presentan signos característicos, como son: hemorragias espontáneas, en mucosas orales y en las articulaciones por algún traumatismo. De acuerdo con datos de la (FMH, 2018) en el 2018 se publicaron cifras del registro Mundial de trastornos de la coagulación sobre más de 1,000 pacientes de 29 Centros de tratamientos de hemofilia y 18 países, los cuales se publican anualmente. Actualmente (FMH, 2019) publicó los resultados del sondeo anual por su 20 aniversario, arrojando que desde 1999 se detectaron 78,629 casos de pacientes con Hemofilia y en el registro del 2019 se obtuvieron 210,454 casos en más de 60 países. En virtud de los resultados se observa que incrementó a $204 \%$ el número de personas con trastornos de la coagulación identificados desde 1999.

Se han desarrollado directrices para el tratamiento odontológico de pacientes con Hemofilia, empleando terapia de remplazo aunado al uso de agentes antifibrinolíticos y hemostáticos locales antes de una cirugía invasora y el bloqueo del nervio alveolar inferior. Por ejemplo, la sutura, aplicación de celulosa oxidizada o goma de fibrina, junto a la administración de concentrado de factor de coagulación. Asimismo, crearon indicaciones de tratamientos de acuerdo con las disciplinas odontológicas por necesidades de tratamiento como, periodoncia, endodoncia, prótesis, procedimientos reconstructivos, cirugía y técnicas de anestesia, sugiere (Brewer et al., 2012). La literatura menciona otros protocolos para el manejo de los pacientes con trastornos de la coagulación. De acuerdo con otras fuentes de consulta se cuenta con guías de práctica clínica en Reino Unido, Colombia, Chile, México, entre otros; relacionadas con el tratamiento estomatológico de niños con Hemofilia A, utilizando medidas alternativas para generar hemostasia en los tratamientos odontológicos, colocación de gomas de fibrina, presión sostenida, uso de láser. Sin excluir las consultas periódicas con su odontólogo, control de higiene bucal para evitar tratamientos invasivos (Grandas Ramírez et al., 2016). Rosario Rodríguez (2017) en Lima-Perú reporta un caso de pulpotomía con electrocauterio en pacientes con Hemofilia A. Utilización de laser quirúrgico odontológico para el control de la hemostasia en los pacientes con trastorno de coagulación (Isidro Olán et al., 2019)

En México, se cuenta con una guía de práctica clínica por parte del Instituto Mexicano del Seguro Social, IMSS para el diagnóstico y tratamiento de la Hemofilia (García Berges et al., 2012) A nivel nacional se han realizados esfuerzos por llevar el factor a los hospitales para mejorar las condiciones de salud de los pacientes con estas características y no han sido suficientes. En Tabasco, existe el Centro de Enseñanza de Hemofilia, dando la atención a esta población junto con un equipo de especialistas en todas las disciplinas de cómo manejar al paciente de manera integral en conjunto con los hospitales. En el caso de la odontología, dentro de las instalaciones del $\mathrm{CEH}$ se cuenta con un consultorio odontológico equipado, donde se realizan tratamientos preventivos y curativos. Todo esto con la finalidad de mejorar las condiciones de salud y calidad de la población con trastornos de la coagulación.

El propósito de este trabajo fue describir el manejo odontológico de pacientes con Hemofilia a través de una serie de casos, donde se pudo realizar procedimientos dentales preventivos y restaurativos empleando medidas alternas de hemostasia y reduciendo la administración de terapia de remplazo.

\section{Material y método}

Investigación de reporte de casos con intervención en pacientes pediátricos con Hemofilia. En el periodo de enero 2019 a agosto 2019, se le pidió la autorización a los pacientes durante este lapso se valoraron 20 pacientes a los cuales se les realizaron 27 procedimientos, con fines para esta investigación, solo se describen los siguientes. Con la siguiente 
metodología, se trabajó con pacientes pediátricos programados a la consulta odontológica del $\mathrm{CEH}$, cuyas edades fluctuaron entre los 0 y 12 años de edad; a quienes se les realizaron tratamientos preventivos y curativos. Utilizando las alternativas de tratamiento para su atención de acuerdo con su severidad y plan de tratamiento a seguir.

\section{Descripción de casos}

\section{Caso 1. Emergencia odontológica}

Paciente S. A. A. masculino de 7 años de edad, diagnosticado con Hemofilia A, moderada (3.2\% de factor circulante en sangre) que acude a consulta en el Centro de Enseñanza de Hemofilia, A.C. por presentar gingivorragia. A la exploración bucal, se observa movilidad dental por exfoliación del OD \#62 y \#63, provocando gingivorragia prolongada con coagulo exofítico de aproximadamente 3(cc) centímetros cúbicos.

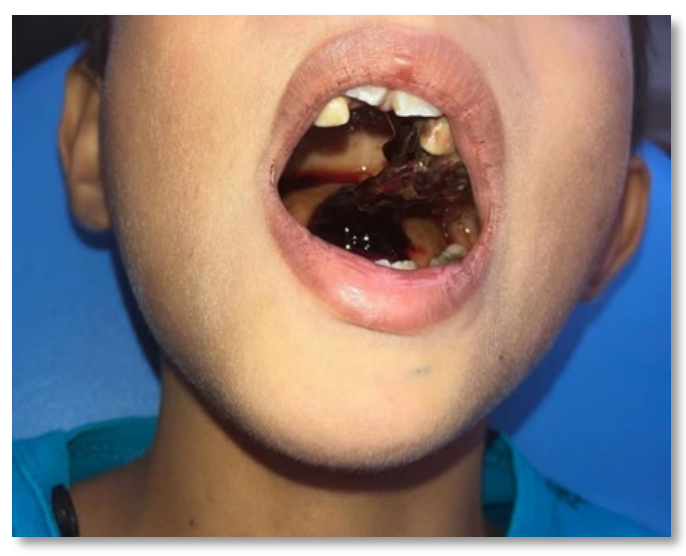

Figura 1. Coágulo exofítico

Plan de tratamiento:

Se administra $500 \mathrm{UI}$ de factor cada $12 \mathrm{hrs}$, por las primeras $24 \mathrm{hrs}$. Calculadas a $20 \mathrm{UI}$ x Kg. Se realiza exodoncia del OD \#62 y \#63. Usando técnica de elevador recto y anestesia local de lidocaína con epinefrina al $2 \%$ con presión sostenida. Con evolución satisfactoria para el caso.

Indicaciones:

- Consulta de control

- Cepillado bucal, las veces que sean necesarias

- Dieta blanda fría

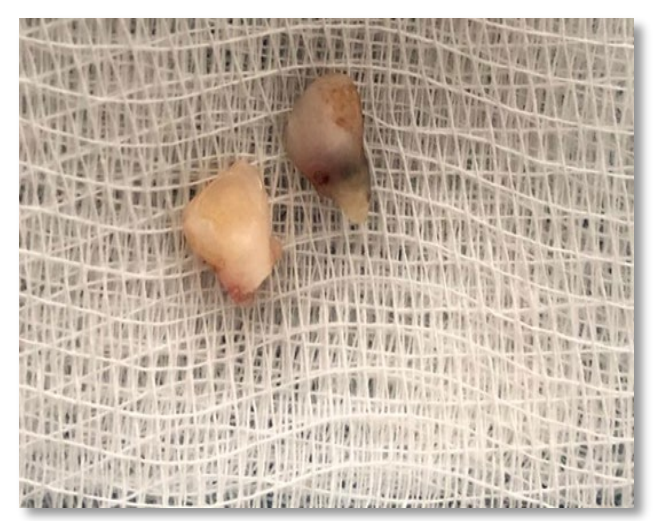

Figura 2. O.D \#62, 63 después de la exodoncia.

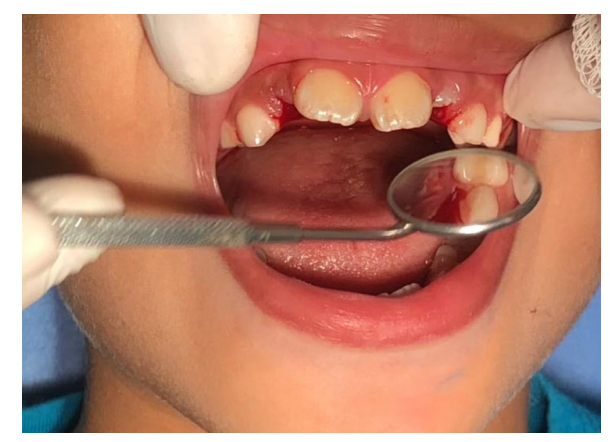

Figura 3. Post operatorio inmediato

\section{Caso 2. Tratamiento con terapia de remplazo y} otras alternativas de hemostasia.

Paciente masculino L.R. de 12 años de edad diagnosticado con Hemofilia A, moderada $(1.4 \%$ de factor circulante en sangre). Acude al Centro de Enseñanza de Hemofilia, A.C. Para su valoración, observando sangrado en capa de manera continua en el OD \#65 por retención prolongada.

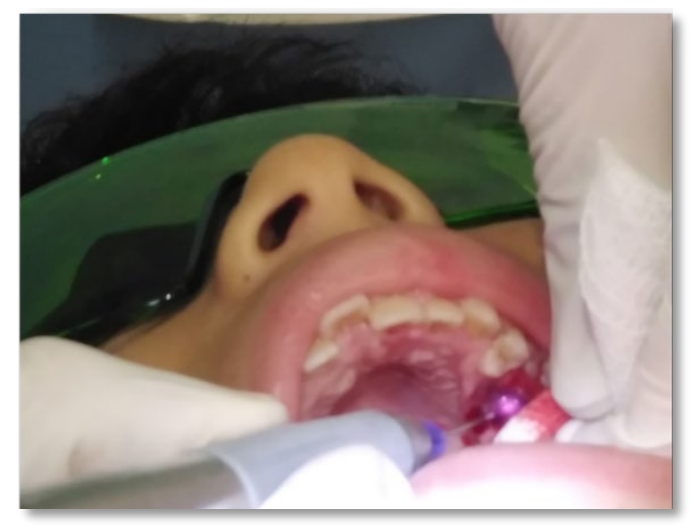

Figura 4. Uso de láser para producir hemostasia. 
Plan de tratamiento:

- Previa administración de factor VIII por profilaxis primaria cada 72 horas a $30 \mathrm{UI}$ x Kg de peso.

- Se realiza exodoncia de OD \#65, con técnica de elevador recto. Después de la extracción para producir hemostasia se utiliza el láser y se coloca sulfato férrico de manera centrípeta en la cara palatina y, por último, se realiza presión sostenida con gasa y agua fría.

- Se corrige dosis a las 24 hrs por sangrado post operatorio a $50 \mathrm{UI}$ cada $12 \mathrm{hrs}$ por 3 días. Con evolución favorable post tratamiento.

Indicaciones:

- Realizar cepillado dental, las veces que sean necesarias.

- Dieta líquida fría.

- Reposo relativo.

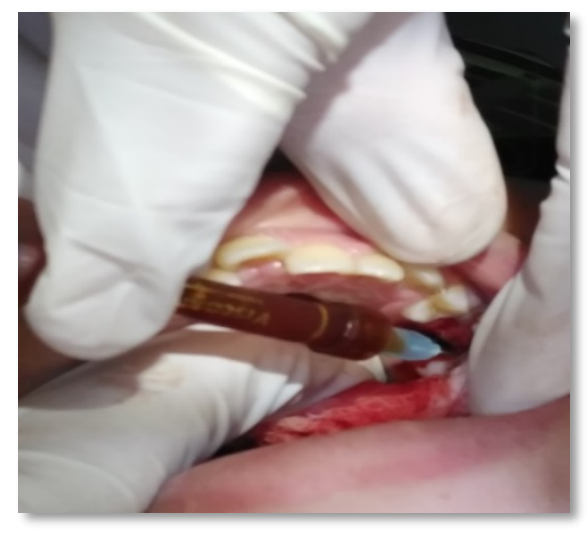

Fiqura 5. Colocación de Sulfato férrico.

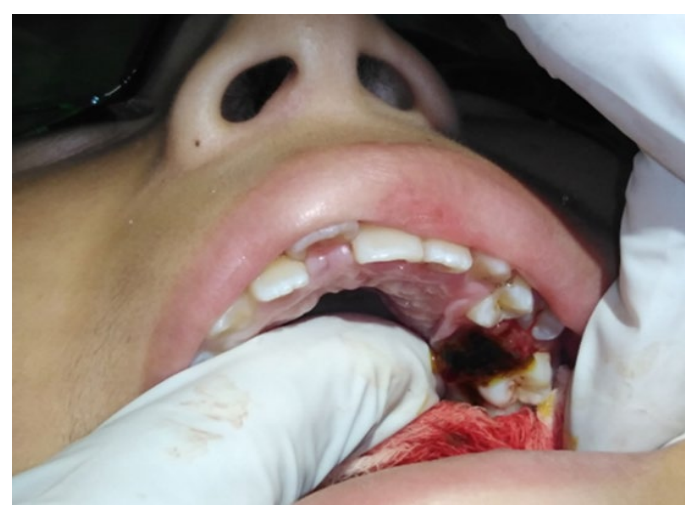

Figura 6. Se aplica presión sostenida con gasa y agua fría.

\section{Caso 3. Control de Higiene Bucodental.}

Paciente masculino A.B.H de 8 años de edad, diagnosticado con Hemofilia A, moderada ( $1.3 \%$ de factor circulante en sangre). Acude al Centro de Enseñanza de Hemofillia, A.C. para su valoración, observa higiene bucal deficiente. Por lo tanto, se le enseña técnica de cepillado al paciente, acorde a sus necesidades.

Plan de tratamiento:

Se realiza control de biofilm con triple plaque, para este procedimiento no se requiere administrar terapia de remplazo.

Indicaciones:

- Emplear correctamente la técnica de cepillado e higiene después de la ingesta de cada alimento.

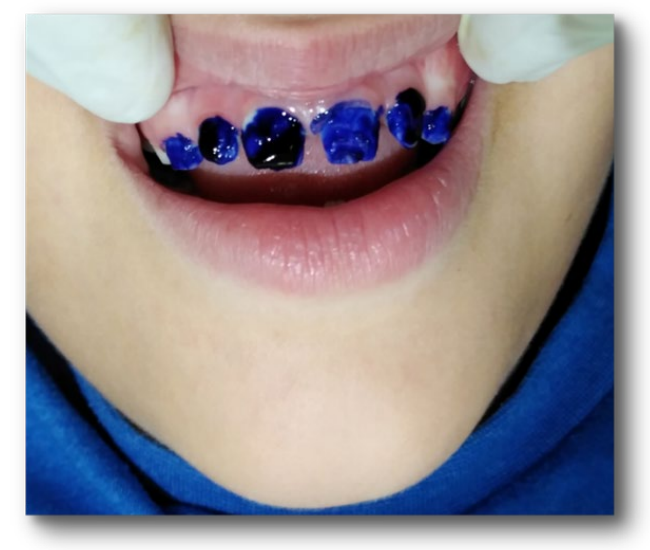

Figura 7. Control de placa dentobacteriana

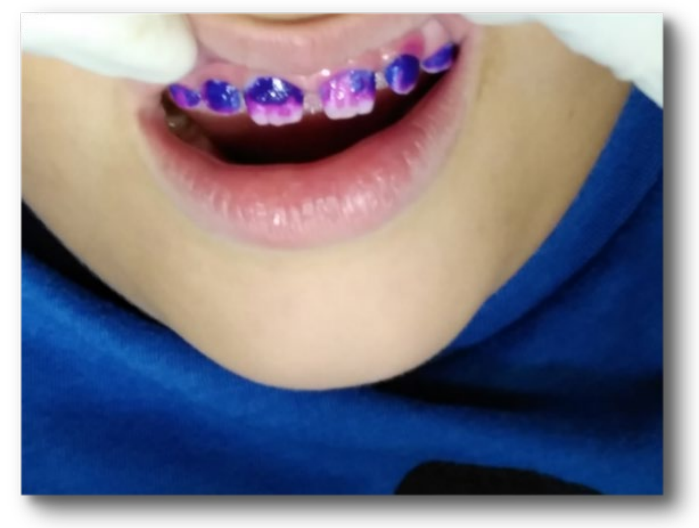

Figura 8. Se observa, en la tinción de color purpura/azul obscuro placa dentobacteriana madura y podemos identificar de color rosa, la placa reciente en la cavidad oral. 


\section{Otros reportes de caso: Tratamientos restaurativos}

\section{Caso 4.}

Paciente masculino $\mathrm{K}$. de 4 años de edad, diagnosticado con Hemofilia B $(2.9 \%$ de factor circulante en sangre) Acude al centro de enseñanza de Hemofilia, A.C. Para su valoración por presentar caries múltiples. A la exploración bucal, se observa mordida abierta anterior y presencia de caries extensas en O.D \#55, 54, 52, 51, 61, 62, 63, 65, 75, 74,84, y 85.

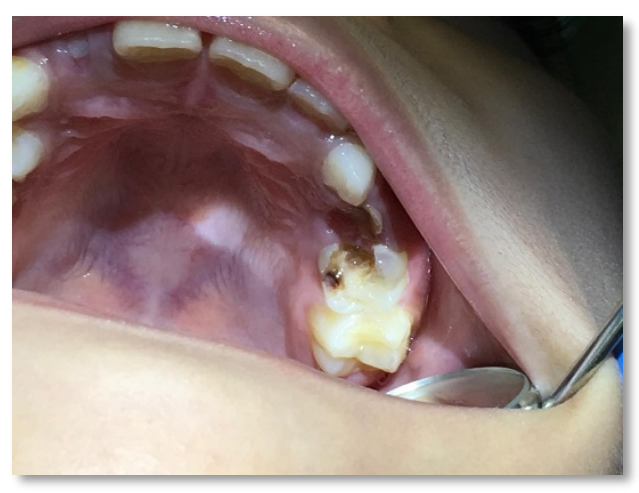

Figura 9. Caries extensa en O.D \#65

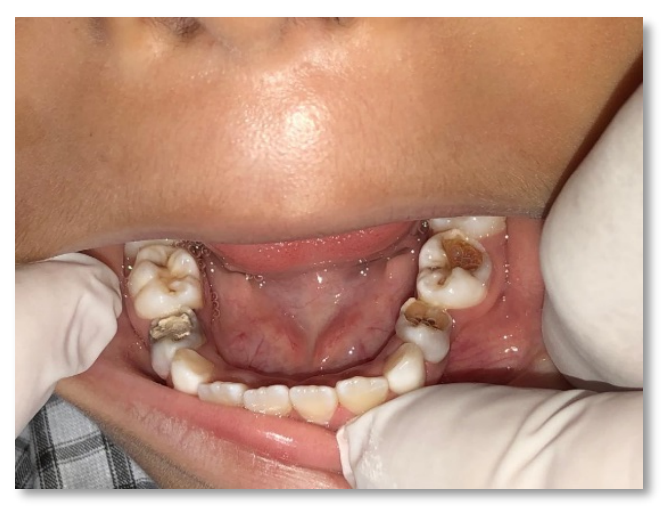

Figura 10. Se observan caries múltiples y restauración temporal con ionómero de vidrio.

Plan de tratamiento:

Se coloca ionómero de vidrio como restauración temporal en los O.D \#75 y \#85. Para este procedimiento no se requiere administrar terapia de remplazo.
Indicaciones:

- Emplear correctamente la técnica de cepillado e higiene después de la ingesta de alimentos.

- Asistir a consultas de control.

\section{Discusión}

Se han evidenciado los diferentes protocolos de atención para el manejo odontológico de pacientes con Hemofilia (Brewer et al., 2006) en los cuales se postula que debe emplearse terapia de remplazo seguida de medidas locales de hemostasia. En caso de ser procedimientos con mínimo de riesgos, propone indicaciones para realizar los tratamientos dentales de prótesis, endodoncia, ortodoncia, técnicas de anestesia y periodoncia. Por lo tanto, en nuestra investigación, se observó que los tratamientos dentales preventivos y curativos pueden ser manejados de manera segura y con mínima invasión, omitiéndose en muchos de los casos la administración de factor deficiente. Si se requiere un procedimiento quirúrgico, se emplea la administración de dosis segura para realizar los tratamientos de acuerdo al peso del paciente en dosis de 8,12 y 24 horas, en base al tipo de procedimiento y riesgo de sangrado post-operatorio.

A nivel mundial, describen guías de práctica clínica 0 protocolos para emplear medidas locales para generar hemostasia en los tratamientos odontológicos; dentro de las cuales se pueden mencionar: la utilización de ácido tranexamico, ácido aminocaproico, gomas de fibrina, presión sostenida y uso de laser (Grandas Ramírez et al., 2016). Tomando en cuenta los protocolos de atención, se puede minimizar la terapia de remplazo acompañado de medidas alternas de hemostasia y uso de medidas hemostáticas locales como presión sostenida, agua fría, hemostáticos locales como, sellador de fibrina, gelfoan, espongstan, láser, uso del sulfato férrico, cianocrilatos, entre otros. De manera similar (Roque González et al., 2006) reporta la utilización de adhesivos tisulares como los cianocrilatos para facilitar los procesos de cicatrización, se encuentran indicados en el área estomatológica y cirugía maxilofacial, en heridas bucales y tratamiento de la estomatitis aftosa.

Otros autores, sugieren que es posible reducir la reposición de factor circulante en sangre. Además, su administración constante puede inducir a la exposición del desarrollo de inhibidores. En los 
tratamientos conservadores, incluso se puede evitar la intervención llevando un buen cuidado oral, atención integral con otras áreas médicas, citas periódicas de control. Educar al paciente para fomentar la prevención de enfermedades periodontales y riesgo de caries. Como señala Quintero Parada (2004) los pacientes con hemofilia son un grupo poblacional vulnerable, por lo tanto, requieren de una atención multidisciplinaria. Tomando en cuenta el padecimiento de cada paciente, se emplean pautas para brindar una atención de calidad, además de la utilización de medidas alternas de hemostasia para reducir la aplicación de terapia de remplazo en los tratamientos odontológicos. Al disminuir la cantidad que se administra, se disminuyen los costos y se reduce la probabilidad de desarrollar inhibidores, beneficiando al paciente y al sistema de Salud. En el Centro de Enseñanza de Hemofilia A.C, se llevan a cabo tratamientos preventivos y curativos.

\section{Conclusión}

El éxito del manejo de pacientes con Hemofilia es brindar una atención odontológica de calidad con el mínimo de riesgos y máximo de beneficios. A pesar de que se cuenta con los protocolos de atención, se puede minimizar la terapia de remplazo acompañado de medidas alternas de hemostasia y uso de medidas hemostáticas locales como presión sostenida, agua fría, hemostáticos locales como: sellador de fibrina, gelfoan, espongstan, láser, uso del sulfato férrico, cianocrilatos, entre otros. Como método de diagnóstico en la valoración dental se utiliza la medición de $\mathrm{pH}$ salival para control de riesgo a caries.

El centro de Enseñanza de Hemofilia A.C, ubicado en Villahermosa, Tabasco. Cuenta con un equipo médico multidisciplinario y dentro de sus instalaciones cuenta con un consultorio odontológico donde se da atención a los pacientes de esta asociación. Existe un programa de atención de la primera infancia de 0 a 3 años y de 3 a 8 años, como grupo prioritario para mejorar las condiciones de salud y adherencia al tratamiento.

A los pacientes con trastornos de coagulación, como es el caso de la Hemofilia, se les pueden realizar tratamientos preventivos y restaurativos sin problemas, disminuyendo los factores de riesgo de sangrado bucal y mejorando su calidad de vida.
No sería posible sin la cooperación del paciente ni el profesional de salud, manejar una atención multidisciplinaria para el control de su salud integral, proporcionando los tratamientos de acuerdo con sus necesidades. Es importante hacer mención que este centro cuenta con capacitaciones mensuales para profesionales de salud y estudiantes; de esta manera pueden aprender el manejo multidisciplinario requerido para la atención de los pacientes con hemofilia

\section{Declaración de conflicto de interés \\ No se reportan conflictos de interés.}

\section{Declaración de fuentes de financiamiento \\ Sin financiamiento y con recursos propios.}

\section{Agradecimientos}

Equipo médico odontológico y a Tabasqueña de hemofilia por todas las facilidades para realizar este trabajo.

\section{Referencias}

Alok Srivastava, Andrew K. Brewer y colaboradores (2012) Guías para el tratamiento de la Hemofilia, 2da edición. Estas guías fueron publicadas originalmente por Blackwell Publishing en Haemophilia: Epub 6 JUL 2012. DOI: 10.1111/j.1365-2516.2012.02909.x y se reimprimen con su autorización.

Bernardoni de Socorro Cecilia, Benito Urdaneta, Marisol, Benito Urdaneta Mariluz, Pereira Granadillo Saúl,Bracho Faria Miriam. (2004) Manejo odontológico del paciente con trastornos hemorrágicos. Una revisión Ciencia Odontológico, de

http://www.redalyc.org/articulo.oa?id=2052221450 07

Brewer Andrew, Correa Elvira Maria. (2006) Directrices para el tratamiento odontológico de pacientes con trastornos de la coagulación hereditarios. Publicado en: eLearning.wfh.org. Por la Federación Mundial de Hemofilia.

Cano Franco Meliza Andrea, Ortiz Orrego Gustavo Eduardo, González Ariza Sandra Elizabeth. (2017). Cuidado odontológico de pacientes con trastornos hereditarios de la coagulación. Revista CES Odontología, 30(1): 
3040.DOl:http://dx.doi.org/10.21615/cesodon.30.1. 3

Federación Mundial de Hemofilia (2019) Sondeo Mundial Anual https://www.wfh.org

Grandas Ramírez, Ángela L. (2016). Niños con hemofilia y su atención odontológica por estomatología pediátrica. Revisión de la literatura / Children with Hemophilia and their Dental Care by Pediatric Stomatology. Review of Literature. Universitas Odontologica, 35(74), 113126. https://doi.org/10.11144/Javeriana.uo3574. nhao

Hermida ML, Álvarez L, Lewis W, Gabriel L, Boggia B, Segovia A, Rodríguez. (2011). Situación de salud oral de niños uruguayos portadores de cogulopatías hereditarias. Centro Hospitalario Pereira Rossell, Monteveido, urugay.

Isidro Olán Laura Beatriz, Rueda Ventura Marco Antonio, Balcazar Najera Carlos Alberto, Najera Castro Armando. (2019) Laser therapy on dental emergencies of patients with hemophilia as an alternative for hemostasis and reducing the use of factor VIII concéntrate. Journal of dental health oral disordes \& therapy.DOl: 10.15406/jdhodt.2019.10.00464

Lorena Bravo, Daniela Muñoz, Consideraciones en el tratamiento odontológico de pacientes pediátricos con Hemofilia. Reporte de un caso clínico. Jornual of oral Research, 2012; 1(2):86-89.

Mannuccio Mannucci Pier. (2012) La desmopresina (DDAVP) en el tratamiento de los trastornos de la coagulación. Federación Mundial de Hemofilia, de www.whh.org .

Quintero Parada E, Sabater Recolons MM, Chimenos Kustner E, López López J. (2004) Hemostasia y tratamiento odontológico. Avances en Odontoestomatología, Vol.20-Núm.5.

Roque González Rosalba, García Gutiérrez Alejandro, Guerra Bretaña Mayelin Rosa, Leal Mursulí Armando, Roque Zambrana Franciso, Cruz Gómez Abigail. (2006). Adhesivos titulares en cirugía. Revista Cubana de Cirugía, 45(3-4).

Rosario Carbonell Rodriguez. Pulpotomía con electrocauterio en pacientes con hemofilia A.reporte. (2017) Universidad Nacional Mayor De San Marcos Universidad Del Perú, Decana De América Facultad De Odontología, Unidad De postgrado.

Salinas Escudero Guillermo,Galindo Suárez Rosa María, Carrillo Vega María Fernanda, Muciño Ortega Emilio. (2013) Análisis del costo y la efectividad de los esquemas de administración de factores de coagulación para el manejo de niños con Hemofilia A en México. Revista Medigraphic, Vol.70.

Recibido: 02 Julio 2019

Aceptado: 30 agosto 2019 